\title{
“Burnout” o Agotamiento en la Práctica Diaria de la Medicina
}

\author{
Douglas Greig Undurraga \\ Instructor Adjunto \\ Departamento de Enfermedades Cardiovasculares \\ $P$. Universidad Católica de Chile \\ Ximena Fuentes Martínez \\ Profesor Asociado Adjunto \\ Departamento de Psiquiatría \\ P. Universidad Católica de Chile
}

Recibido el 30 de octubre de 2009, aceptado el 27 de noviembre de 2009

Rev Chil Cardiol 2009; 28: 403-407

"Para Hernán, residente de primer año de cardiología, las cosas no estaban siendo fáciles. Hernán, fue un alumno destacado en su pregrado y el comienzo de esta nueva etapa era para el un logro y una aspiración, por lo cual la inició con entusiasmo. Paulatinamente se vio con cada vez más responsabilidades en el cuidado de sus pacientes, exigencias en los aspectos docentes y definiciones en su vida personal. Inicialmente, pensó que era lógico estar cansado, pero se dio cuenta que le costaba concentrarse en sus estudios y retener lo aprendido; además sentía a los pacientes y familiares muy exigentes y demandantes. Lo comentó con sus compañeros, pero todos le dijeron que era normal, que se le iba a pasar, así es que no le dio mayor importancia y se dispuso a superarlo. Pero con el tiempo notó que ir al hospital sólo significaba hacer rápido el trabajo en la sala y, además, sus compañeros más cercanos notaron el hecho de verlo menos dedicado y perder la preocupación por el cuidado de sus pacientes. Concomitantemente, sus profesores lo presionaban a rendir más y a escribir más trabajos. Hernán, ya no podía más, se sentía cansado y llegó a pensar en retirarse de la especialidad, creyendo que no era capaz, que a lo mejor no tenía las condiciones y que en la cardiología, a pesar que le seguía interesando, ya no veía la posibilidad de alcanzar un logro personal".

\section{Introducción al Problema}

El tema del efecto del estrés sobre las personas que trabajan con personas, es motivo de reflexión y estudio desde los años 70 . Muchos de los profesionales dedicados al cuidado de otros, están siendo considerados profesionales en riesgo de enfermar en el ejercicio de su quehacer, a este síndrome se le denomina Burnout.

El Burnout, que en español puede ser traducido como "agotamiento" -"fundirse", "quemarse"- se ha descrito como "la respuesta patológica a estresores emocionales e interpersonales en el contexto del trabajo diario"1.

El síndrome de desgaste profesional o burnout se presentaría en personas altamente motivadas por su trabajo, idealistas y con deseos de entregar mucho de si, que se ven sobrepasados por las condiciones laborales y el nivel de demanda personal al que se ve sometido, en relación a las gratificaciones que recibiría en el desempeño de su rol1.

En los últimos decenios se ha experimentado un cambio en la forma como la sociedad visualiza

Correspondencia: Dr. Douglas Greig Undurraga

Departamento de Enfermedades Cardiovasculares.

Pontificia Universidad Católica de Chile. Marcoleta $367,7^{\circ}$ piso.

Unidad Coronaria - Santiago

Teléfono: 02-3543624 - Fax: 02-3543644

Correo Electrónico: dgreig@puc.cl 
la relación del individuo con el trabajo, los modelos considerados válidos son cada día más competitivos e individualistas, centrados en el logro y el éxito, lo cual impone una sobre exigencia al individuo, la de ser exitoso laboralmente desde el punto de vista de estos referentes.

Lo anterior favorece la aparición de síntomas de estrés en los individuos, quienes además de la exigencia propia de su labor clínica, deben responder a múltiples demandas de un nivel distinto al técnico (eg. empatía, comprensión y dedicación), horarios exigentes y turnos; es decir, demandas agregadas a la excelencia clínica, que de no ser percibidas y manejadas en forma apropiada por el individuo, pueden desencadenar burnout.

Este síndrome, fue descrito inicialmente en el campo de la psicología ocupacional, afectando a aquellas personas en que tenían un grado mayor de relación interpersonal. Por este motivo, se entiende que es un problema actual y que involucra, especialmente, a personas vinculadas a la salud. Según Maslach, el burnout, "Es un síndrome de agotamiento emocional, despersonalización y baja realización personal que puede ocurrir entre individuos que trabajan con personas" e involucra tres dimensiones: agotamiento o cansancio emocional, despersonalización y realización personal. El cansancio emocional se define como la pérdida progresiva de las energías vitales y desproporción entre el cansancio experimentado y el trabajo realizado. El individuo presenta una mayor irritabilidad y quejas por la cantidad de trabajo, con sensación de gran esfuerzo, tanto físico como psicológico ("no doy más"). La despersonalización es el elemento clave del burnout y se caracteriza por la pérdida de preocupación por el paciente y respuestas distantes y cínicas hacia las personas a las que se les presta servicio, incluido los propios colegas. Por otra parte, la falta de realización personal se caracteriza por la autoevaluación negativa del propio trabajo. Esta falta de "logro personal", se refiera al sentimiento que tiene la persona cuando se siente menos competente en la resolución de su trabajo1,2.

En la medida que el individuo es sometido a distintos estresores a lo largo de su desempeño vital, va desarrollando actitudes de adaptación y formas de enfrentarlo, lo que ha sido descrito por Seyle como síndrome de adaptación general, el cual explica la respuesta del organismo al estar sometido a un estresor crónico. Las etapas de este síndrome son: Alarma, Resistencia y Agotamiento.

De acuerdo a esto, Etzion plantea que el burnout es un proceso latente de erosión psicológica, resultante de una exposición prolongada al estrés laboral, cuya última fase, el agotamiento, aparecería sin que el individuo haya percibido las fases previas ${ }^{3}$.

\section{Población de estudio y magnitud del problema}

El problema del burnout ha sido estudiado en varias poblaciones en el ámbito médico. Se ha descrito en grupos de enfermeras, médicos staff e incluso en Decanos de escuelas de medicina. Al respecto, un estudio de Gabbe et $\mathrm{al}^{4}$, señala una prevalencia de hasta un $4 \%$ de burnout en Decanos de Escuelas de Medicina Norteamericanas. Sin embargo, y dado la importancia que reviste en el proceso educativo, nos referiremos al subgrupo de médicos residentes. Según diversos estudios en este subgrupo, la prevalencia varía desde $18 \%$ hasta $82 \%$, siendo su promedio en aquellos trabajos bien realizados en alrededor de $40 \% 1,2,5$. La gran diferencia en las prevalencias se puede explicar por las diferentes metodologías empleadas para su diagnóstico, así como en el sesgo que tienen este tipo de estudio en determinar el problema en los residentes con menos disponibilidad de tiempo y que, eventualmente, podrían tener una prevalencia mayor. Con respecto a los exámenes utilizados para su diagnóstico, el más utilizado es el "Maslasch Burnout Inventory (MBI)", el cual, mide en una escala del 0 al 6 el grado de compromiso en las tres dimensiones mencionadas.

Dado que el estrés tiene un rol importante en el desencadenamiento y evolución de trastornos mentales. El residente con burnout tiene una mayor probabilidad de padecer de otras patologías, principalmente, trastornos del ánimo, tendencias al pensamiento psicótico, depresión psicótica, somatización, distimia, abuso de alcohol y drogas ilícitas y trastornos de ansiedad generalizada1.

Al respecto, un interesante estudio publicado por Dyrbie et al 6 mostró que en un grupo de estudiantes de medicina, la prevalencia de burnout fue de 
aproximadamente $50 \%$ y que un $10 \%$, tuvieron ideación suicida durante alguna etapa de su carrera. El autor mostró que los estudiantes con burnout se asociaron significativamente a la ideación suicida y que la intervención con el fin de disminuir la prevalencia de burnout, se asoció igual disminución en la ideación suicida. Por otro lado, el residente con burnout se ha considerado como un riesgo ocupacional. Diversos estudios han reportado una mayor tasas de incidentes y manejo subóptimo del paciente 1 .

\section{Factores de riesgo asociados y problemas específicos}

Los factores de riesgo se pueden dividir en ocupacionales (organizacionales) e individuales.

Los factores de riesgo ocupacionales han sido ampliamente estudiados; sin embargo, sus resultados no han sido muy consistentes. Un reciente estudio que evaluó la importancia del "ambiente de trabajo", mostró que en 3.080 estudiantes de medicina norteamericanos, el clima de aprendizaje negativo, dado por factores tales como ausencia de un ambiente de trabajo colaborativo, de rotaciones que promueven el aprendizaje o el apoyo de autoridades, entre otros, se asoció de manera significativa con la prevalencia de burnout (OR 1,36$2,07, p \leq 0,02)$. Este mismo estudio, reportó una mayor prevalencia de burnout en aquellos estudiantes que tienen turnos nocturnos; pero, en forma interesante, mostró que la carga de trabajo (eg. número de ingresos) no se asocia a una mayor prevalencia de burnout7. Esto puede explicarse, ya que al residente no le preocupa la alta carga laboral a la cual se ve enfrentado, sino que más bien, el "ambiente" como se enfrenta dicha carga o "la falta de control" de la carga laboral que se le ha impuesto.

Otros factores que se han asociado a la presencia de burnout son: la "percepción estresante" del trabajo, problemas financieros asociados a éstos, los conflictos asociados al trabajo en el hogar, grado de participación en las decisiones (a mayor participación se reduce el riesgo y aumenta la satisfacción laboral), las largas jornadas de trabajo, la intensidad requerida en este (número de personas atendidas y/o duración del contacto con el paciente), disponibilidad y uso de soportes sociales (ie. retroalimentación con los compañeros de trabajo) y la ambigüedad de rol, conflictos de rol y expectativas poco realistas sobre el profesional1.

Con respecto a los factores de riesgo individuales, se ha reportado una mayor prevalencia de burnout en hombres, raza blanca, residentes solteros, conflictos familiares, desempleo o trabajos "part-time" del cónyuge, entre otros. ${ }^{1,2}$. Además, las personas idealistas, optimistas e ingenuas, que se entregan de forma inconciente demasiado al trabajo para tener una buena opinión de sí mismas, están en riesgo de tener burnout.

Otros factores de riesgo, estadísticamente menos significativos, pero no menos importantes son la subespecialidad (médicas versus quirúrgicas), las horas de sueño, el bajo nivel de autonomía en la toma de decisiones, la auto-duda constante, el "papeleo" excesivo, el perfeccionismo, el uso de drogas o tabaco, falta de tiempo para el autocuidado, dificultad con pacientes y familiares complejos, entre otros ${ }^{1,5}$.

Si bien es cierto, no existen claras diferencias entre las especialidades, éstas afectan diferentes dimensiones. De este modo, se ha descrito una mayor prevalencia de despersonalización en las especialidades quirúrgicas y de intensivos a diferencia de especialidades con mayor contacto familiar o ambulatorio.

Al contrario, diversos estudios han mostrado que el uso de terapias de relajación, grupos de soporte por parte de médicos entrenados ("counseling"), "mentores" en el caso de médicos con mayores responsabilidades (staff jóvenes) ${ }^{8}$, uso y disponibilidad de dinero, mayor control sobre rotaciones y horarios, entre otros, se han asociado a una menor prevalencia de burnout. Del mismo modo, los programas de educación médica continua ( $\mathrm{CME}$, por sus siglas en inglés) se han asociado a una menor prevalencia de "stress laboral" y burnout. Esto puede deberse a que hay una mejor percepción de "logro personal" 9 .

\section{Reflexión final y recomendaciones}

El burnout es un problema preocupantemente prevalente en nuestro medio y que afecta al estudiante profundamente. Este lo compromete en el plano personal, laboral, en su desempeño con sus pacientes y en su hogar. 
El burnout es un proceso que se desarrolla gradualmente en el tiempo, producto de las tensiones por las discrepancias entre las expectativas y las demandas al que el individuo se ve sometido, especialmente en el ámbito laboral -"un proceso gradual de pérdida de energía durante el cual el desajuste entre las necesidades de la persona y las demandas del trabajo crecen cada vez más"10- éste se presenta en individuos sin sicopatología previa y que se han desempeñado previamente bien; puede provocar agotamiento emocional, físico, psicológico, disminución del compromiso con el trabajo y baja en su funcionamiento general previo.

Como se mencionó, el buen clima de aprendizaje y de trabajo es fundamental en la prevención del burnout. Es tarea de las organizaciones cuidar que aquellas condiciones laborales que facilitan la aparición del mismo se modifiquen y que se disminuyan así los factores de riesgo. Por otro lado, el burnout atenta contra la comprensión y retención de conocimientos, así como contra la promoción del autoaprendizaje, que es uno de los principales legados que podemos dejar en nuestros estudiantes.

Algunos factores protectores que dependen del ámbito ocupacional u organizacional son los siguientes:

- Crear condiciones de trabajo que transmitan preocupación, cuidado y seguridad.

- Crear condiciones de catarsis en los equipos. Estructurarlos en reuniones técnicas.

- Compartir la responsabilidad de las decisiones.

- Establecer condiciones de confianza en los equipos (reuniones no laborales relajadas, rituales de incorporación y de despedida).

- Estilos de liderazgo democráticos (no autoritarios).

- Estilos de supervisión fortalecedores de los propios recursos.

- Resolución de conflictos no confrontacionales.

- Apoyo de la institución para la formación continua.

- Comunidad ideológica, marco teórico compartido, o cultura organizacional común a todos los miembros.

- Activación y fortalecimiento de las redes de apoyo, consultorías profesionales.

- Generación de instancias de descompresión: seminarios, talleres de capacitación, encuentros profesionales.

Por otro lado, existen tareas que el propio individuo puede desarrollar para su autocuidado de una manera conciente:
- Hacerse cargo de uno mismo y de su cuidado personal.

- Registro oportuno y visibilización de los malestares: registrar las necesidades básicas, sed, hambre, cansancio, dolores.

- Registrar las molestias psicológicas, lo que permite enfrentar los conflictos oportunamente.

- Vaciamiento y descompresión de los contenidos de fuerte impacto emocional: compartir con otros, entre pares, personas que comprendan y contengan.

- Mantención de áreas libres de contaminación: tener espacios de actividades absolutamente libres de las temáticas del trabajo ("hacer algo por el placer de hacerlo").

- Evitar saturar las redes personales de apoyo.

- No contaminar los espacios personales, pareja, hijos y amigos con contenidos laborales. Cuidar relaciones amorosas y darles un tiempo real.

- Formación profesional: Es una exigencia propia de la tarea habitual y que protege del burnout. Recibir formación en aquellas perspectivas teóricas y modelos que entreguen destrezas instrumentales adecuadas.

- Ubicar la responsabilidad donde corresponde: Evitar autoresponsabilizarse en exceso y evitar culpar a otros.

- Desarrollo de la espiritualidad: Confiar y creer en algo superior a uno mismo.

En nuestra Escuela de Medicina se realiza con éxito hace 2 años, un curso transversal de "Técnicas de Prevención del Burnout", orientado a residentes. En este curso, se enseñan técnicas de relajación, imaginería y medicación, junto con contenidos cognitivos acerca del estrés y el burnout. Estas estrategias de autocuidado deben ser conscientes y de esta manera, pueden ser aprendidas por el residente.

En este sentido, el instaurar éstas estrategias de prevención y otras tales como la lectura, la música, terapias de grupo, terapias de autoayuda basadas en la WEB, "counseling", "mentorías" y otros 11-13, son indispensables en la formación íntegra de nuestros futuros profesionales. La responsabilidad es de todos nosotros. 


\section{Agradecimientos}

Dr. Carlos Reyes, del Depto. de Medicina Interna de la P. Universidad Católica y Docente del curso "Residentes Clínicos Como Docentes".

\section{Referencias}

1. JELLE TP, STACEY MG-D, BEN JT, FRANK MMA, HARRY BM, JOSETTE EHMH. Burnout in medical residents: a review. Medical Education. 2007; 41: 788-800.

2. THOMAS NK. Resident burnout. JAMA. 2004; 292: 2880-2889.

3. ETZION D. Moderating effect of social support on the stress-burnout relationship. J Appl Psychol. 1984; 69: 615-22.

4. GABBE SG, WEBB LE, MOORE DE, HARRELL FE JR, SPICKARD WA JR, POWELL R JR. Burnout in medical school deans: an uncommon problem.

Acad Med. 2008; 83: 476-82.

5. ECKLEBERRY-HUNT J, LICK D, BOURA J, HUNT R, BALASUBRAMANIAM M, MULHEM E, et al. An exploratory study of resident burnout and wellness.

Acad Med. 2009; 84: 269-77.

6. DYRBYE LN, THOMAS MR, MASSIE FS, POWER DV, EACKER A, HARPER W, et al. Burnout and suicidal ideation among U.S. medical students. Ann Intern Med. 2008; 149: 334-41.

7. LISELOTTE ND, MATTHEW RT, WILLIAM H, MASSIE JR FS, DAVID VP, ANNE E, et al. The learning environment and medical student burnout: a multicentre study. Medical Education. 2009; 43: 274-82.
8. GABBE SG, WEBB LE, MOORE DE JR, MANDEL LS, MELVILLE JL, SPICKARD WA JR. Can mentors prevent and reduce burnout in new chairs of departments of obstetrics and gynecology: results from a prospective, randomized pilot study. Am J Obstet Gynecol. 2008; 198: 653-57.

9. TALMA K, AVNER HC, ELI K. Continuing medical education and primary physicians' job stress, burnout and dissatisfaction. Medical Education. 2000; 34: 430-436.

10. MASLACH C, SCHAUFELI WB, LEITER MP. Job burnout. Annu Rev Psychol. 2001; 52: 397-422.

11. LANDI L, REGOLI G, ROVERSI A, MITELLO L. Therapeutical rehabilitation project and burnout: a experience of quali-quantitative research in a psychiatric department]. Prof Inferm. 2008; 61: 112-19.

12. VAN STRATEN A, CUIJPERS P, SMITS N. Effectiveness of a web-based self-help intervention for symptoms of depression, anxiety, and stress: randomized controlled trial. J Med Internet Res. 2008; 10:e7.

13. MACKERETH PA, WHITE K, CAWTHORN A, LYNCH B. Improving stressful working lives: complementary therapies, counselling and clinical supervision for staff. Eur $\mathrm{J}$ Oncol Nurs. 2005; 9: 147-54. 